\title{
La escritura académica en los procesos de formación en investigación: representaciones, saberes y contrastes"
}

\author{
Academic writing in the research formation process: \\ representations, knowledge and contrasts
}

Yury Andrea Castro Robles" y Humberto Sánchez Rueda"

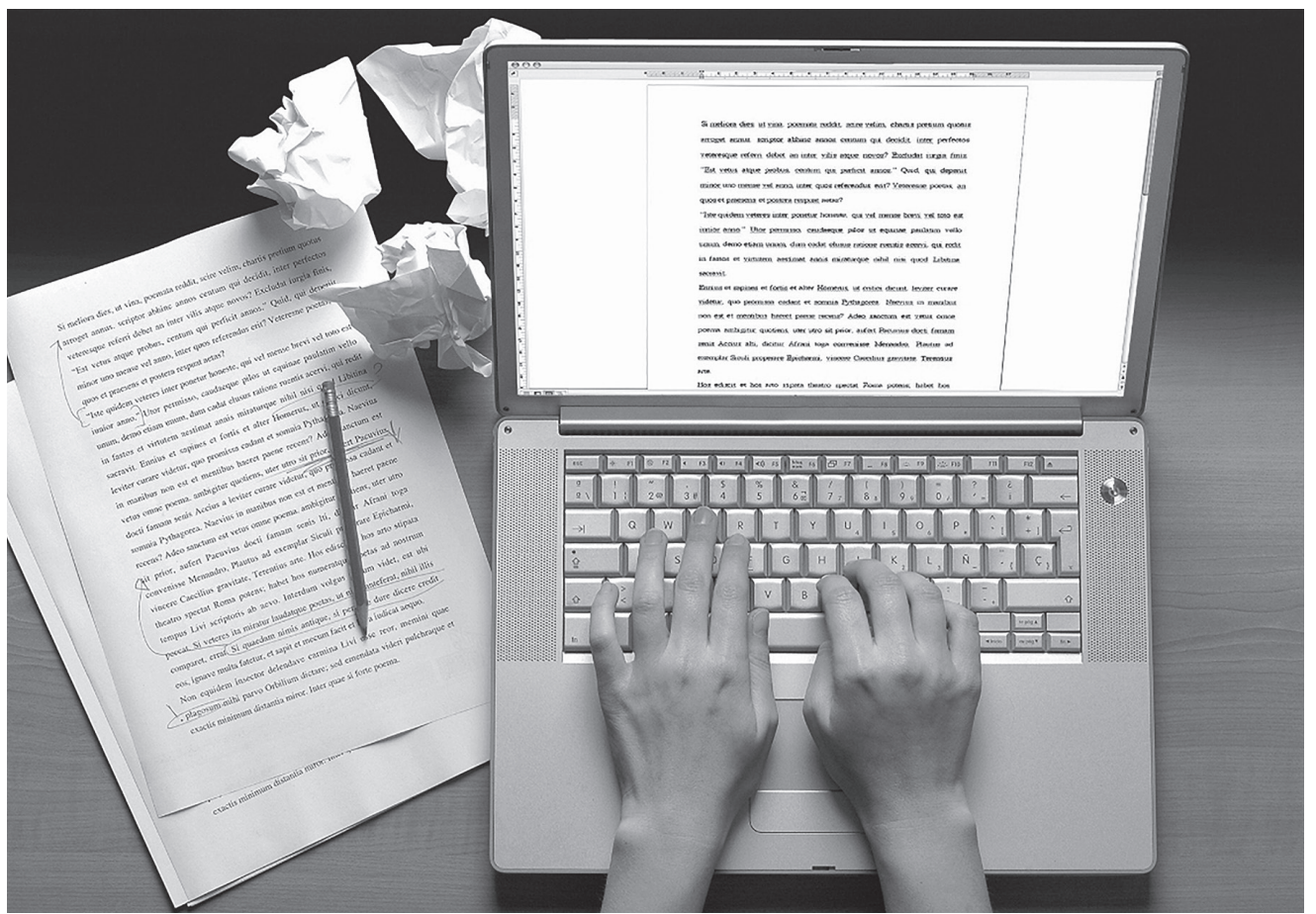

Citar este artículo como: Castro Robles, Y. y Sánchez Rueda, H. (2016). La escritura académica en los procesos de formación en investigación: representaciones, saberes y contrastes. Revista Papeles, 8(15), pp. 21-36.

Fecha de recibido: marzo 15 de 2016.

Fecha de aceptación: 30 de junio de 2016

Este artículo constituye un avance de la indagación acerca de las prácticas de escritura en los procesos de enseñanza y aprendizaje en el programa de Licenciatura en Lengua Castellana e Inglés de la Universidad Antonio Nariño, que se está realizando desde 2015-02 y se encuentra programado hasta 2016-02.

* Docente de la Facultad de Educación de la Universidad Antonio Nariño. Magíster en Filosofía de la Pontificia Universidad Javeriana y Licenciada en Humanidades y Lengua Castellana de la Universidad Distrital Francisco José de Caldas. Correo electrónico: yurcastro@uan.edu.co

** Profesional en Estudios Literarios de la Universidad Nacional de Colombia y Magíster en Educación con énfasis en la enseñanza de la lectoescritura y matemáticas de la Universidad Externado de Colombia. Ha publicado artículos sobre crítica literaria y formación docente. Actualmente es profesor de la Facultad de Educación de la Universidad Antonio Nariño. Correo electrónico: hsanchezr@uan.edu.co 
La contradicción es, en verdad, entre la escritura y la obra (el Texto, a su vez, es una palabra magnánima: no toma en cuenta esta diferencia). Gozo sin solución de continuidad, sin fin, sin término, de la escritura como de una producción perpetua, una dispersión incondicional, una energía de seducción a la que ninguna defensa legal del sujeto que echo sobre la página puede ya detener. Pero en nuestra sociedad mercantil hay que llegar a la postre de una "obra": hay que construir, es decir, terminar una mercancía. Así, mientras escribo, la escritura se ve en todo momento aplanada, banalizada, culpabilizada por la obra a la cual se ve obligada a contribuir

(...) La escritura es ese juego en el cual me doy vuelta más o menos bien dentro de un espacio demasiado estrecho: estoy arrinconado, me debato entre la histeria necesaria para escribir y el imaginario, que vigila, amanera, purifica, banaliza, codifica, corrige, impone la mira (y la visión) de una comunicación social. Por un lado quiero que me deseen y por el otro que no me deseen: histérico y obsesivo al mismo

tiempo.

Barthes (1978, p. 149)

\title{
Resumen
}

En las últimas décadas, la discusión sobre la escritura en el ámbito académico ha privilegiado el estudio de las prácticas académicas, las situaciones didácticas, los factores socioculturales y los déficits discursivos de los estudiantes. En contraste, este artículo busca contribuir a dicha reflexión desde la indagación y cuestionamiento del saber común (representaciones sociales) en torno a la escritura en los procesos de formación en investigación que tienen estudiantes y docentes, en este caso específico, en la realización de los trabajos de grado en la Licenciatura en Lengua Castellana e Inglés de la Universidad Antonio Nariño. Tal indagación reconoce que la heterogeneidad de las culturas académicas también se configura desde los saberes y las creencias de sus participantes, lo que permite la identificación de las correspondencias y divergencias entre saber común y pensamiento científico sobre la escritura en los procesos de formación en investigación.

Palabras clave: escritura académica, investigación, representaciones, enseñanza, universidad.

\begin{abstract}
During the last decades, the discussion of the writing process, in the academic field, has focused on the study of academic practices, didactic situations, sociocultural factors and students' discursive deficit. In contrast, this article aims to contribute to this reflection from the search and controversy of common knowledge (social representations) in relation to writing inside the research formation process of students and teachers, in this particular case, in the execution of degree projects in Bachelor Degree in Spanish Language and English at Antonio Nariño University. Such inquiry recognizes that heterogeneity of academic cultures is also configured from the participants' knowledge and beliefs, allowing the identification of correspondences and differences between common knowledge and scientific thinking about writing inside the research formation process.
\end{abstract}

Keywords: academic writing, research, representations, teaching, university. 


\section{Situación inicial: escribir en los procesos de investigación de pregrado}

En la actualidad, como lo han señalado los estudios de Jurado (1998), Carlino (2003, 2004a, 2004c, 2005a, 2006), Arnoux et al. (2004), Arnoux (2006), Ortiz (2015), entre otros, la escritura en los procesos de investigación en pregrado, principalmente, se concibe como el medio que permite registrar y transmitir el saber producto de las indagaciones investigativas realizadas por los estudiantes. En estos contextos, la escritura aparece en las etapas finales de la formación en investigación con el propósito de dar a conocer a la comunidad académica productos obtenidos. Desde esta perspectiva, la escritura tiene una función comunicativa esencialmente. Se presenta, no como una actividad constante y regular, sino como un elemento al final del proceso de investigación. Este entendimiento de la escritura como una práctica de la inmediatez, como un producto, hace que los procesos en relación a ella se agoten en sus aspectos formales simplificando sus posibilidades conceptuales y temáticas.

A pesar de que la universidad considera que el estudiante no ha consolidado sus procesos escritores al llegar a la educación superior y en su discurso hace un llamado al fortalecimiento de estos procesos, apenas proporciona unas pequeñas pautas fragmentadas para el desarrollo de la escritura y responsabiliza de esta labor al estudiante (Pérez y Rincón, 2013). Las universidades ofertan cursos, especialmente al inicio de los programas académicos, con los que buscan nivelar a los estudiantes en sus competencias. Sin embargo, tales espacios, como se evidencia en la investigación realizada por REDLEES (Red de Lectura y Escritura en la Educación Superior) en 2015 (González y Salazar, 2015), cuentan con poco tiempo, reducen la escritura a un problema gramatical y sus didácticas separan la escritura de los procesos de aprendizaje propios de cada disciplina.
En tales contextos, no existe un reconocimiento de las posibilidades epistémicas de la escritura en los procesos investigativos, en palabras de Jurado (1998), no se piensa la formación en investigación en relación al "acto de pensar desde la interrogación y la incertidumbre" (p. 69) que genera el acto de escribir. Los estudiantes no encuentran en los espacios ofrecidos por las universidades la posibilidad de reflexionar su práctica investigativa desde la escritura, de "interpretar su quehacer" (Jurado, 1998, p. 69) a través de la palabra escrita. Por el contrario, conciben la escritura como un "ejercicio aislado" al final del periodo académico, que da cuenta de los resultados del proceso. Al no concebirse la escritura como un elemento que permite la organización del pensamiento, el estudiante pierde la posibilidad de ordenar y re-significar sus procesos cognitivos. En este sentido, como lo señala van Dijk en entrevista realizada en el 2015, la escritura entendida como discurso precisa de la comprensión no solo de una estructura, "también se necesita una dimensión cognitiva (sobre los procesos mentales de la producción y de la comprensión del discurso)" (Ferrer, 2015, p. 11).

Estas dificultades entre escritura e investigación se agudizan en el momento final de los estudios universitarios, donde se le exige al estudiante dar cuenta, a través de la escritura, de la experiencia investigativa final realizada como requisito de grado. El "trabajo de grado" (mal llamado tesis en el pregrado en algunos contextos) se convierte en un "dolor de cabeza" para el estudiante y su realización en "la piedra en el zapato" que no permite conceptualizar y sintetizar el ejercicio investigativo realizado.

Las anteriores circunstancias, acerca de la función de la escritura en las propuestas de formación en investigación en el pregrado, acusan contrastes y heterogeneidades en tres elementos principalmente: concepción, 
Tabla 1. Dificultades de la escritura en los procesos de investigación en pregrado

\begin{tabular}{lll}
\hline \multicolumn{1}{c}{ Concepción de escritura } & \multicolumn{1}{c}{ Prácticas de escritura } & \multicolumn{1}{c}{ Escritura e investigación } \\
\hline Registrar y transmitir información & $\begin{array}{l}\text { Escritura al final de los procesos } \\
\text { investigativos }\end{array}$ & $\begin{array}{l}\text { La investigación se condensa en la } \\
\text { escritura de un trabajo de grado }\end{array}$ \\
\hline $\begin{array}{l}\text { Desconocimientos de posibilidades } \\
\text { epistémicas de la escritura }\end{array}$ & $\begin{array}{l}\text { La escritura se entiende como } \\
\text { un bien adquirido en la escuela }\end{array}$ & $\begin{array}{l}\text { Separación del proceso de investiga- } \\
\text { ción y escritura }\end{array}$ \\
\hline $\begin{array}{l}\text { Propósitos diferentes en la } \\
\text { evaluación }\end{array}$ & $\begin{array}{l}\text { No existen experiencias de } \\
\text { reflexión de escritura académica }\end{array}$ & $\begin{array}{l}\text { Dificultades para escribir los resulta- } \\
\text { dos de los procesos de investigación }\end{array}$ \\
\hline
\end{tabular}

Fuente: elaboración propia

prácticas y función en la investigación (resumen en la tabla 1 ):

Ante este panorama, se pueden formular hipótesis de estudio que permiten delimitar caminos de indagación para repensar las posibilidades de la escritura en los procesos de formación en investigación en los programas académicos de educación. En primer lugar, se evidencia la separación de la escritura del proceso de investigación, por lo cual se dificulta la formación de un docente con actitud investigativa. Como lo ha señalado Jurado (1998), el investigador auténtico solo aparece en el momento "en que se atreve a registrar lo observado, cuando privilegia el dato auténtico y cuando organiza a través de la escritura de las conjeturas; cuando ubicado en ese campo de la práctica pedagógica, describe e

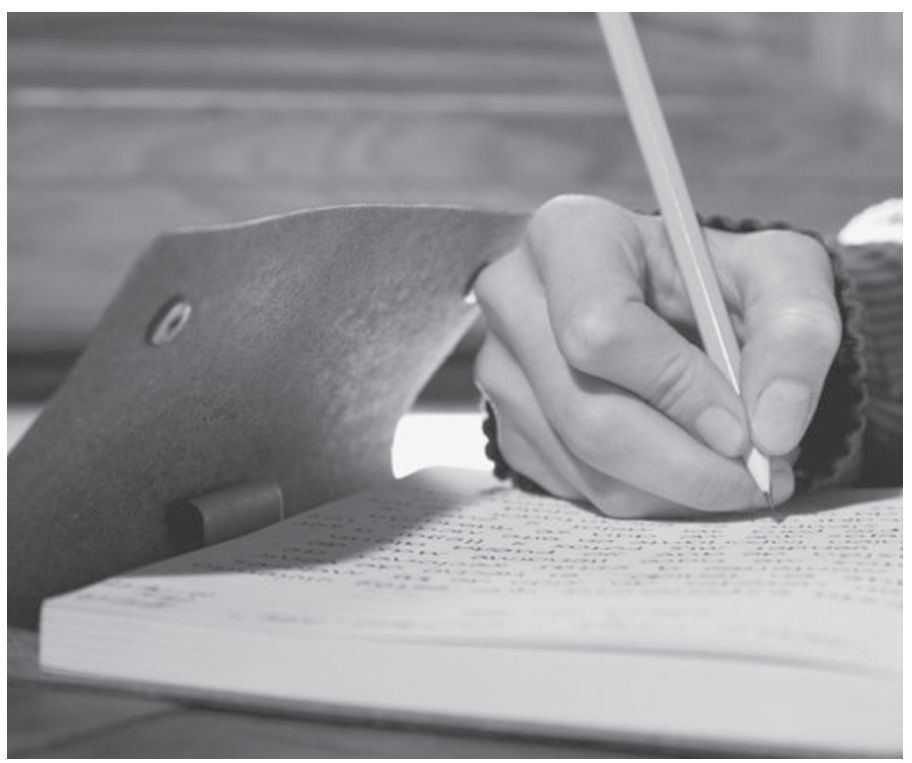

interpreta lo que ocurre" (p. 71). La escritura permite entonces que la experiencia investigativa se convierta en material de reflexión y de producción de conocimiento nuevo; sin ella ningún proceso investigativo podría cumplir sus objetivos. En este sentido, el papel de las prácticas de escritura en la investigación resulta fundamental en tanto permite que "la investigación formativa, en efecto, aborde el problema de la investigación como aprendizaje de la investigación misma y en este sentido el rol del docente sería el de fomentar una cultura investigativa mediada por las acciones" (Saby, 2012, p. 26). Ante los contrastes de las relaciones entre formación docente, escritura e investigación, resalta que las universidades conciben la importancia de la formación en investigación, sin tener claro el papel de la escritura académica en tales procesos.

En síntesis, la separación entre las prácticas de escritura académica y los procesos investigativos dificultan, en muchos casos, la posibilidad de que los estudiantes reconozcan la escritura académica como una herramienta que permite ordenar y conceptualizar el ejercicio investigativo. En este marco, se propone reflexionar y analizar las prácticas de escritura académica en la realización de trabajos de grado de los estudiantes de la Licenciatura en Lengua Castellana e Inglés (LLCI) de la Universidad Antonio Nariño (UAN), a fin de ofrecer un marco descriptivo que permita delimitar puntos de partida para resignificar las posibilidades de la escritura en los procesos de formación en investigación. 


\section{Punto de partida para la reflexión: las representaciones sociales de la escritura en los procesos de investigación}

Sobre las causas de la anterior situación, investigadores como Arnoux (2006), Carlino (2003, 2004b, 2005a, 2005b, 2013), Pérez y Rincón (2013), entre otros, han encontrado importantes puntos de discusión en las prácticas académicas, las situaciones didácticas, los factores socioculturales y los déficits discursivos que los estudiantes han heredado de la escuela. En los resultados de estas investigaciones sobresale el hecho de que en las universidades tanto los estudiantes y maestros como los documentos institucionales reconocen el papel fundamental de la escritura en los procesos académicos; sin embargo, existe heterogeneidad respecto a la concepción, procesos y finalidades de la escritura en la educación superior. Es así, que de cierta forma, no existe un panorama claro que permita entender la relación entre escritura e investigación en la formación integral de los estudiantes de acuerdo a sus disciplinas, contextos e individualidades. Por tanto, se propone que el estudio de la concepción y las funciones de la escritura en la universidad, y en este caso en los procesos investigativos, puede partir del reconocimiento de las creencias y las concepciones que los sujetos (estudiantes y maestros) tienen de la escritura. Como señala Carlino (2004a), desde estas creencias, se configuran perspectivas que motivan acciones, promueven deseos y describen el mundo (ya sea de forma consciente o inconsciente), por lo cual desde su reconocimiento y estudio se puede contribuir al diseño de alternativas que permitan potencializar las posibilidades de la escritura en la universidad.

Estas concepciones y creencias desde la psicología social francesa, en cabeza de Sergei Moscovici y de Dennis Jodelet, han sido denominadas representaciones sociales. En 1969 Moscovici definió el concepto de representación social en el prefacio que realizó a la obra de Herzlich, titulada "Santé et Maladie: analyse d'une représentation sociales" (citado en Farr, 1985), como un "sistema de valores, nociones y prácticas que proporciona a los individuos los medios para orientarse en el contexto social" (p. 497), esto es, "un corpus organizado de conocimientos y una de las actividades psíquicas gracias a los cuales los hombres hacen inteligibles la realidad física y social, se integran a un grupo o en una relación cotidiana de intercambios" (p. 497).

Se trata del reconocimiento de que las acciones y los deseos de los sujetos son socialmente compartidos, por lo cual el pensamiento científico confluye con el saber del sentido común y determina el ser social de las personas. En conclusión, señala Jodelet (1985), las representaciones sociales "designan una forma de conocimiento específico, del saber de sentido común, cuyos contenidos manifiestan la operación de procesos generativos y funcionales socialmente caracterizados. En sentido más amplio, designa una forma de pensamiento social" (p. 475).

En este sentido, el estudio de las representaciones sociales de la escritura en los procesos de elaboración de trabajos de grado permite proponer bases conceptuales que lleven a formular estrategias efectivas $y$ acordes a las necesidades de los contextos educativos, que potencialicen el papel epistémico de la escritura en los procesos de investigación. Esto contribuiría satisfactoriamente a la formación integral de los estudiantes, cualificar las escrituras de los trabajos de investigación y contribuir desde la escritura a los procesos cognitivos desarrollados en la investigación. De este modo la universidad "debe proponerse como el lugar idóneo donde dichas representaciones deben ser puestas en discusión, a partir de la mirada epistémica de la especialización pedagógica" (Sánchez, 2013, p. 95), contribuyendo significativamente a los procesos de aprendizaje de los estudiantes.

Revista PAPELES • ISSN 0123-0670 • Vol. 8(15) • pp. 21-36 • Enero-junio de 2016 


\section{Ruta para la identificación de las representaciones sociales de la escritura en la investigación en la LLCI de la UAN}

Desde la anterior perspectiva, se busca identificar las representaciones sociales de la escritura en la investigación en el programa de LLCI de la UAN, a partir de una indagación interpretativa que está orientada a describir e interpretar fenómenos privilegiando el estudio de los significados e intenciones de las conductas humanas (variables no observables) desde el punto de vista de los propios agentes educativos (Erickson, 1989). Por tanto, se busca comprender las problemáticas señaladas entre escritura e investigación desde la cotidianidad y las opiniones dadas por los agentes educativos que participan en la situación. De esta forma, se privilegia una investigación interpretativa desde el enfoque metodológico de la teoría fundamentada (Grounded Theory), ya que se propone una metodología de análisis unida a la recolección de datos, que utiliza un conjunto de métodos, sistemáticamente aplicados, para generar una teoría inductiva sobre un área sustantiva. De esta forma, los objetivos de la indagación constituyen una formulación teórica, o un conjunto integrado de hipótesis conceptuales, sobre el área substantiva que es objeto de estudio (Glaser, 1992).

Desde el enfoque de la teoría fundamentada, la identificación de las representaciones sociales de escritura de trabajos de grado en el programa LLCI de la UAN se propone en tres etapas que buscan recolectar, interpretar y proponer a partir de la información seleccionada. A continuación se resumen las etapas de la ruta investigativa en la Tabla 2:

En un momento inicial, se realizó una indagación teórica acerca de textos que señalan las relaciones entre representaciones sociales y escritura. Para el caso latinoamericano, los textos de Carlino (2004a, 2005a), Arnoux (2006) y Moya et al. (2013) hacen referencia explícita a las representaciones sociales de la escritura. A partir de estas referencias junto a la conceptualización sobre representaciones sociales, alfabetización, escritura académica e investigación, se delimitaron dos referentes de análisis para pensar las representaciones sociales de la escritura en relación con la escritura de trabajos de grado: (1) representación social de la escritura y (2) representaciones de la escritura en los procesos de investigación. Asimismo, se identificaron componentes e indicadores en cada categoría que permitían delimitar la formulación de las representaciones sociales de la escritura, como se precisa en la siguiente tabla:

Tabla 2. Etapas de la ruta metodológica

\begin{tabular}{lll}
\hline No. & \multicolumn{1}{c}{ Etapa } & \multicolumn{1}{c}{ Tipo de actividades } \\
\hline 1 & $\begin{array}{l}\text { Diseño y validación de } \\
\text { técnicas de recolección de } \\
\text { información. }\end{array}$ & $\begin{array}{l}\text { Formulación de categorías de análisis. } \\
\text { Diseño de encuestas. } \\
\text { Revisión de técnicas requeridas de acuerdo a la perspectiva del trabajo. }\end{array}$ \\
\hline 2 & $\begin{array}{l}\text { Recolección y análisis de la } \\
\text { información documental } \\
\text { desde categorías de análisis } \\
\text { (Referentes). }\end{array}$ & $\begin{array}{l}\text { Aplicación de técnicas de recolección de información. } \\
\text { Recolección de información documental institucional relacionada con la } \\
\text { escritura. }\end{array}$ \\
\hline 3 & $\begin{array}{l}\text { Caracterización de la } \\
\text { información y conclusiones. }\end{array}$ & $\begin{array}{l}\text { Interpretación de la información recolectada a partir de las categorías de } \\
\text { análisis desde los conjuntos de información seleccionada. }\end{array}$ \\
\hline
\end{tabular}

Fuente: elaboración propia 
Tabla 3. Componentes e indicadores en el estudio de las representaciones sociales de la escritura

\begin{tabular}{lll}
\hline \multicolumn{1}{c}{ Categoría } & \multicolumn{1}{c}{ Componentes } & \multicolumn{1}{c}{ Indicadores } \\
\hline $\begin{array}{l}\text { Representación } \\
\text { social de la } \\
\text { escritura }\end{array}$ & $\begin{array}{l}\text { Concepto de escritura } \\
\text { Función social de la } \\
\text { escritura }\end{array}$ & $\begin{array}{l}\text { Reconoce qué es la escritura en el saber del sentido común. } \\
\text { Comprende para qué sirve la escritura desde la perspectiva del } \\
\text { Conocimiento común. }\end{array}$ \\
& $\begin{array}{l}\text { Contextos escriturales } \\
\text { Gusto por la escritura }\end{array}$ & $\begin{array}{l}\text { Identifica cuáles son los métodos aceptados socialmente para } \\
\text { escribir. }\end{array}$ \\
& $\begin{array}{l}\text { Reconoce en qué ámbitos de la vida social se necesita escribir. } \\
\text { Siente gusto por el acto de escribir. }\end{array}$ \\
\hline $\begin{array}{l}\text { Representaciones } \\
\text { sociales de la } \\
\text { escritura en } \\
\text { los procesos de } \\
\text { investigación }\end{array}$ & $\begin{array}{l}\text { Investigador como } \\
\text { escritor }\end{array}$ & $\begin{array}{l}\text { Reconoce que a partir del registro y reflexión de la información } \\
\text { producto de la investigación aparece la figura del investigador. }\end{array}$ \\
& Escritura epistémica & Escribe para dar cuenta del conocimiento producto de la \\
& investigativa & Comprende que en la investigación la escritura permite \\
& Momentos de la & diseñar y reestructurar el conocimiento. \\
& escritura & $\begin{array}{l}\text { Reconoce la necesidad de escribir durante los distintos } \\
\text { momentos del proceso investigativo (antes, durante, después). }\end{array}$ \\
& Escritura privada y & Identifica que en los procesos de investigación existen prácti- \\
& escritura pública & cas de escritura privadas y públicas. \\
\hline
\end{tabular}

Fuente: elaboración propia

Teniendo en cuenta estas categorías y sus componentes, se establecieron dos ejes de recolección de información: los documentos institucionales y el pensamiento sobre escritura e investigación de estudiantes y docentes. Ante estas fuentes, se propusieron las técnicas más acordes para recopilar la mayor cantidad de información posible, teniendo en cuenta las categorías señaladas: lectura de documentos institucionales (Proyecto Educativo de Programa (PEP), syllabus de asignaturas relacionadas con la realización de trabajos de grado y Práctica pedagógica I, II y III) y encuestas semiestructuradas.

Por un lado, la lectura de documentos institucionales permite identificar las concepciones fundantes del programa LLCI en torno a las relaciones entre escritura e investigación, evidenciando la existencia de referencias explícitas de los componentes de las categorías señaladas (tabla 3). Tales referencias permiten delimitar las apuestas institucionales acerca del lugar de la escritura en los procesos de aprendizaje en el programa. Hay que tener en cuenta en la lectura de estos documentos, que el no señalamiento explícito de la información a indagar, no permite la realización de conclusiones, sino más bien la formulación de hipótesis que permitan reflexionar sobre tal situación.

Y por otro, para indagar sobre el pensamiento de docentes y estudiantes, se proponen dos encuestas semiestructuradas, una para cada de población. Este tipo de encuesta permite identificar con un componente de preguntas y respuestas fijas tendencias dominantes en situaciones de escritura cotidianas en la academia y, a la vez, con una serie de preguntas abiertas se puede reconocer, de acuerdo con Lucca y Berrios (2003), el comportamiento complejo de los participantes sin limitarlos a categorizaciones realizadas por el investigador. Las preguntas de la encuestas se construyeron a partir de los indicadores de los componentes de las categorías, por lo cual se propone una encuesta para docentes con 23 preguntas (17 cerradas y 6 abiertas) y otra para estudiantes con 15 preguntas (10 cerradas y 5 abiertas). 
La encuesta para estudiantes estuvo dirigida a todos aquellos que estaban realizando su trabajo de grado en el período 2015-2 en LLCI y fue respondida por 17 estudiantes. Igualmente, la encuesta de profesores se dirigió a un grupo del equipo docente que estaba relacionado con la elaboración de los trabajos de grado (ya sea como tutores, jurados, profesores de cursos de investigación), siendo respondida por 7 docentes. La encuesta tenía una duración aproximada de 15 a 20 minutos y no solicitaba el nombre de la persona que la diligencia, para así invitar a la objetividad y claridad de las respuestas.

\section{Resultados parciales: identificación e interpretación de las representaciones sociales de la escritura en la investigación en el programa LLCI}

A continuación se presentan los resultados parciales del análisis de la información recolectada en las encuestas y los documentos institucionales, de acuerdo a las dos categorías señaladas. No obstante, debido a la cantidad de información, solo se presentarán algunos puntos del análisis desde la referencia a los componentes propuestos en las categorías.

\section{Categoría 1: Representaciones sociales de la escritura}

Con el objetivo de identificar y analizar las representaciones sociales de la escritura en el programa de LLCI, se propusieron cinco componentes que permiten reflexionar sobre los sistemas de pensamientos sociales que se tienen sobre la escritura: concepción, función, metodologías, contextos y sentimientos (gusto), como se especificó en la tabla 3. A continuación se presentan las primeras aproximaciones.

\section{Concepción y función de la escritura para docentes y estudiantes}

En el programa de LLCI, la enseñanza y aprendizaje de la escritura es un eje fundamental de formación debido a que uno de los propósitos de sus futuros egresados constituye la formación de "sólidos conocimientos lingüísticos, didácticos y pedagógicos, así como una alta competencia comunicativa en español e inglés" (UAN, PEP, 40). En los programas de formación docente que tienen como saber disciplinar el estudio del lenguaje, la escritura adquiere una importancia fundamental en el desarrollo de las competencias comunicativas y los campos conceptuales de la lingüística. No obstante, en muchos casos, no se considera necesario explicitar cómo la escritura participa de este proceso de modo constante en la formación de los estudiantes. Asimismo, en los syllabus (contenidos programáticos) de los cursos de línea temática más cerca de la escritura académica (lingüística, literatura, producción textual), se señala la importancia de la escritura en la formación académica sin evidenciar orientaciones o situaciones didácticas que permiten su aprendizaje. En contraste, en el apartado de los syllabus denominado "Criterios de evaluación", se hace una mención distinta a la escritura, ya que se indican nombres de tipologías y géneros textuales en torno a porcentajes de evaluación cuantitativa, debido a que en la actual cultura académica la escritura es el medio predilecto para evaluar el aprendizaje, por encima de producciones orales o audiovisuales.

Al contraponer tales resultados con las indagaciones realizadas con estudiantes y docentes, la heterogeneidad respecto a las concepciones de escritura aumenta al entenderse como un medio de transmitir información, principalmente, como se resume en la tabla 4.

A pesar de la aparente variedad de respuestas resalta el reconocimiento explícito de 
Tabla 4. Concepciones y funciones de la escritura para estudiantes y docentes de la UAN

\begin{tabular}{|c|c|c|}
\hline Preg & Estudi & Docentes \\
\hline $\begin{array}{l}\text { ¿Para usted } \\
\text { qué significa } \\
\text { escribir? }\end{array}$ & $\begin{array}{l}\text { Comunicar ideas } \\
\text { Un proceso personal y reflexivo } \\
\text { Una actividad rigurosa de expresión del pensamiento } \\
\text { Una manera de significar el mundo } \\
\text { Una facultad de representaciones de ideas } \\
\text { Una práctica que implica lecturas previas } \\
\text { Un arte } \\
\text { Un proceso } \\
\text { Dar cuenta de sus capacidades lingüísticas } \\
\text { Reconocerse como sujetos } \\
\text { Transmitir cultura } \\
\text { Ser un medio de expresión del pensamiento } \\
\text { Dar sentido a las cosas } \\
\text { La acción de transmitir ideas, sentimientos y experiencias } \\
\text { Escribir a alguien } \\
\text { Buscar precisión y claridad en lo que se plantea o se propone } \\
\text { Dar cuenta de lo que se sabe }\end{array}$ & $\begin{array}{l}\text { Un proceso de organización del } \\
\text { pensamiento } \\
\text { Es un medio de expresión } \\
\text { Es un arte y una técnica } \\
\text { Transmitir ideas } \\
\text { Redactar algo de una manera } \\
\text { ordenada y coherente } \\
\text { Expresar lo que sentimos } \\
\text { Reconocer el estado de sus } \\
\text { competencias comunicativas }\end{array}$ \\
\hline $\begin{array}{l}\text { ¿Qué tan } \\
\text { importante } \\
\text { considera la } \\
\text { escritura? }\end{array}$ & $\begin{array}{l}\text { Es un medio de comunicación del pensamiento } \\
\text { Ayuda a sintetizar lo que se quiere decir } \\
\text { Ayuda a comprender el mundo } \\
\text { Exige pensar y comprender lo que se dice } \\
\text { En ella yace el conocimiento de la cultura humana. } \\
\text { Importante en la educación } \\
\text { Fundamental en los procesos de evaluación } \\
\text { Muy importante } \\
\text { No mucho, es una actividad académica } \\
\text { Ayuda a mostrar lo que se aprende en la universidad } \\
\text { Importante en la formación profesional } \\
\text { Permite mostrar lo que se sabe y siente } \\
\text { No es necesaria } \\
\text { Bastante, ya que ayuda a la evaluación } \\
\text { Muy difícil, pero importante } \\
\text { Contribuye al trabajo de la escuela y universidad } \\
\text { Normal, igual que otros procesos }\end{array}$ & $\begin{array}{l}\text { Es una necesidad comunicativa } \\
\text { humana } \\
\text { Es un proceso de reflexión e } \\
\text { investigación que conlleva a escribir } \\
\text { con claridad las ideas de alguien } \\
\text { Es un medio de comunicación } \\
\text { directo } \\
\text { Fortalece la memoria y la vida } \\
\text { humana } \\
\text { Da cuenta de sus competencias } \\
\text { comunicativas } \\
\text { Es una herramienta de expresión } \\
\text { académica } \\
\text { Muy importante para dar cuenta } \\
\text { del conocimiento }\end{array}$ \\
\hline
\end{tabular}

Fuente: elaboración propia

la importancia de la escritura en el conocimiento. Sin embargo, de esta indicación, se evidencia la falta de consenso acerca de la concepción y el papel que cumple la escritura en los encuestados. Sigue siendo la capacidad de transmitir información la noción más clara que tienen la mayoría de encuestados sobre la función de la escritura (14 de 17 respuestas de estudiantes, y 4 respuestas de 7 de docentes); en cambio, en unos pocos casos se hace mención al reconocimiento de las funciones epistémicas y cognitivas de la escritura. En conclusión, existe la creencia compartida acerca de la importancia de la escritura en la comunidad académica (documentos, docentes y estudiantes) y el papel fundamental de la escritura en la transmisión de la información, aunque al mismo tiempo, tal importancia responde a una heterogeneidad de componentes.

\section{Dónde y cómo escriben estudiantes y profesores}

Si la heterogeneidad es la característica más llamativa de la concepción y las funciones de la escritura en los estudiantes y profesores, de igual forma sucede en lo que respecta a los 
contextos y metodologías de escritura. Por un lado, podríamos señalar que tal diversidad obedece a los distintos contextos sociales, familiares y culturales de formación a los que pertenecen los integrantes de la comunidad académica. Y, por otro, se desconoce esta variedad en los procesos de aprendizaje al pensarse la universidad como un espacio homogéneo donde todos sus integrantes tienen los mismos intereses.

Ante las preguntas con opción múltiple que buscan indagar por los espacios en que se escribe, los estudiantes respondieron: tiempo de ocio (41\%), vida privada ( $23 \%$ ), redes sociales $(64 \%)$, materias ( $46 \%$ ) y trabajos (53\%). En cambio, las respuestas de los docentes fueron: estudios de posgrado (60\%), vida privada ( 80 $\%)$ y labores académicas (100 \%). A diferencia de los estudiantes, la cotidianidad de los docentes está atravesada por la escritura.

Con el objetivo de delimitar las metodologías que se utilizan para escribir, a través de una pregunta abierta se interrogó a los estudiantes acerca de las metodologías y elementos que intervienen en los procesos escriturales. Vale la pena señalar que los estudiantes no tienen métodos recurrentes para escribir. Por ejemplo, una estudiante ante la pregunta “ ¿tiene alguna forma específica para empezar a escribir?", señala lo siguiente: "hago como pintor en el lienzo y dejo que las ideas salgan libremente". Esta respuesta sirve para explicar la espontaneidad con la que se asume la actividad escritora, sin reconocer procesos conceptuales y metodológicos que permiten elaborar de mejor forma ciertos tipos de textos en la academia. Tal aserción no significa que, en ciertos momentos de la escritura, la libre expresión no se considere importante; se trata de que este no puede ser el único elemento para asumir los procesos escriturales.

A pesar de que no se reconocen herramientas conceptuales o metodologías que permitan la mejor realización de los textos escritos diferentes a la espontaneidad y la inspiración; esto contrasta con el reconocimiento de la necesidad de estas herramientas en el momento de examinar las dificultades de sus escritos. Vemos entonces, que ante la pregunta "cuando elabora un texto ¿qué elementos de este proceso le parecen difíciles?", se encuentra que el $76 \%$ considera difícil mantener la coherencia del texto; y en segundo lugar, un 47 $\%$ señala el uso adecuado de los signos de puntuación. Al contrastar estos dos resultados, se hace evidente el reconocimiento que se tiene de elementos semánticos y sintácticos del texto en contraste con el desconocimiento de los elementos de los procesos de comprensión y producción textual.

En relación a los profesores, se les preguntó por los elementos que se privilegian en los métodos de enseñanza de la escritura y resalta el porcentaje obtenido en la opción de "enseñanza de las reglas gramaticales" (70\%). En las prácticas docentes sigue siendo una constante el énfasis por la gramática, quizás como ha señalado Lerner (2001), porque es lo más evidente en el proceso inicial de evaluación. No obstante, opciones como la coherencia y el sentido comunicativo, se consideran importantes por un alto sector (60\%). Quizá estas diferencias en los porcentajes se deben a las dificultades que en la práctica tiene pensar las relaciones entre los componentes gramaticales, semánticos y comunicativos. En estos casos el instrumento es limitado, e invita a revisar las prácticas cotidianas de enseñanza de la escritura.

\section{Sentimientos que genera la actividad de escribir}

Finalmente, el sentir deseo por escribir constituye también un punto fundamental en las representaciones sociales de la escritura debido a que las actividades en las que se reconoce gusto y aprecio, a pesar de su dificultad, generan vivencias significativas que permiten un mejor aprendizaje. Como lo afirma Zuleta (1982), la "escritura es aventura, el 'sentido' es múltiple, irreductible a un querer decir, irrecuperable, inapropiable, 'nadie puede escuchar en 
las cosas' (...) Se carece de oídos para escuchar aquello a que no se tiene acceso desde la vivencia” (p. 10). Desde esta perspectiva, se indaga por los sentimientos que genera la escritura desde seis opciones de respuesta (gusto, disfrute, satisfacción, frustración, apatía, angustia, motivación), donde se podía marcar más de una opción. Los resultados más altos fueron gusto y satisfacción con un $52 \%$, pero a la vez, frustración y angustia tenían porcentajes altos con $42 \%$ y $38 \%$ respectivamente. Se puede inferir que frente a la escritura existe un sentimiento de ambivalencia; a los estudiantes les gusta escribir, pero al mismo tiempo les genera sentimientos difíciles. Aunque el instrumento no alcanza para revisar esta contraposición, se puede arriesgar a proponer que como actividad privada la escritura genera satisfacción; y quizá en el momento cuando se vuelve pública, cuando tiene un evaluador, la angustia y la frustración invaden al escritor.

\section{Categoría 2: Representaciones sociales de la escritura en la investigación}

El sistema de creencias compartidas que existe sobre la escritura académica en el programa de LLCI permite pensar de mejor forma las relaciones entre escritura e investigación. Es así que las características que se han identificado en la concepción, componentes, funciones y prácticas constituyen las bases de la relación entre escritura e investigación, y más precisamente, en el momento que los estudiantes escriben sus trabajos de grado. A partir de los componentes señalados, se proponen tres ejes para sistematizar las representaciones sociales de la escritura en los procesos de investigación.

\section{Investigador como escritor}

En la encuesta realizada se les preguntó a los estudiantes " ¿cuál considera es el papel de la escritura en los procesos investigativos?", para indagar las relaciones entre la figura del investigador y el escritor a partir de 4 opciones: (a) Descripción del proceso investigativo (63 \%), (b) Sistematizar experiencias (10\%), (c) Comunicar algo (19\%) y (d) Producir conocimiento (8\%).

Las tendencias con mayor acogida (a y c) identifican al investigador como un escritor que registra la información obtenida. Sin duda, esta labor constituye una de las principales funciones de la escritura en la investigación, ya que permite que la información permanezca, y pueda ser comunicada. De cierta forma, se puede agregar que si el investigador no escribe, su investigación prácticamente no existe. En contraste, muy pocos estudiantes (la tendencia es muy baja) consideran que la reflexión de la información producto de la investigación es algo importante en la investigación. Sin duda esta percepción es heredera del papel tan preponderante que tiene la función comunicativa de la escritura, desconociendo sus posibilidades epistémicas. Asimismo, los tipos de texto que los estudiantes han privilegiado en la escritura de su investigación en su mayoría han sido textos de divulgación de la información (documentos finales, presentaciones en Power Point) y en menor cantidad textos que contribuyen a reflexionar la información recogida (comentarios, diarios de campo, talleres, ponencias, foros), como se aprecia en la tabla 5:

Esta misma tendencia se repite en buena parte en las prioridades que tienen los profesores asesores de trabajos de grado, ya que privilegian la escritura del documento final de presentación, debido, por un lado, a que este es el que permitirá dar a conocer la investigación de los estudiantes y, por otro, los tiempos tan cortos para acompañar la realización de los escritos:

En conclusión, en la escritura de trabajos de grado se reconoce al investigador como un escritor, ya que registra y comunica los productos de la investigación. No obstante, no se entiende el binomio escritor-investigador como alguien que reflexiona la información obtenida. 
Tabla 5. Prácticas de escritura en la realización de trabajos de grado

\begin{tabular}{lrrrr}
\hline & Todos los días & Cada semana & Cada Mes & Cada Corte \\
\hline Toma de apuntes & $47,62 \%(10)$ & $52,38 \%(11)$ & $0 \%(0)$ & $0 \%(0)$ \\
\hline $\begin{array}{l}\text { Comentarios por escrito o fichas de lectura de } \\
\text { bibliografía secundaria }\end{array}$ & $7,69 \%(1)$ & $76,92 \%(10)$ & $15,38 \%(2)$ & $0 \%(0)$ \\
\hline Reseñas & $20 \%(2)$ & $30 \%(3)$ & $30 \%(3)$ & $20 \%(2)$ \\
\hline Ensayos & $13,33 \%(2)$ & $20 \% 83)$ & $40 \%(6)$ & $26,67 \%(4)$ \\
\hline Talleres & $16,67 \%(2)$ & $66,67 \%(8)$ & $16,67 \%(2)$ & $0 \%(0)$ \\
\hline Ponencias & $0 \%(0)$ & $25 \%(2)$ & $37,5 \%(3)$ & $37,5 \%(3)$ \\
\hline Presentaciones en Power Point, carteleras & $0 \%(0)$ & $38,46 \%(5)$ & $30,77 \%(4)$ & $30,77 \%(4)$ \\
\hline Foros virtuales, blog & $0 \%(0)$ & $0 \%(0)$ & $25 \%(2)$ & $75 \%(6)$ \\
\hline Escritura del documento final & $12,5 \%(2)$ & $18,75 \%(3)$ & $12,5 \%(2)$ & $56.25 \%(9)$ \\
\hline Otro & $0 \%(0)$ & $0 \%(0)$ & $0 \%(0)$ & $100 \%(4)$ \\
\hline
\end{tabular}

Fuente: elaboración propia

Tabla 6. Actividades realizadas por los profesores asesores

\begin{tabular}{lr}
\hline $\begin{array}{l}\text { Propuso una pauta o guía que permitía } \\
\text { planificar el escrito }\end{array}$ & $14,29 \%$ \\
$\begin{array}{l}\text { Solicitó avances del escrito para su } \\
\text { calificación }\end{array}$ & $28,75 \%$ \\
$\begin{array}{l}\text { Solicitó avances del escrito para su } \\
\text { retroalimentación, independiente de }\end{array}$ & \\
$\begin{array}{l}\text { que fuera o no calificado } \\
\text { Asesoró la escritura }\end{array}$ & $85,71 \%$ \\
$\begin{array}{l}\text { Solo recibió el producto final para su } \\
\text { calificación }\end{array}$ & $100 \%$ \\
Otro & $14,29 \%$ \\
\hline
\end{tabular}

Fuente: elaboración propia

\section{Comunicación de conocimiento frente a escritura epistémica investigativa}

Las distintas respuestas de los estudiantes respecto a los procesos, evaluaciones y momentos de la escritura de sus trabajos de grado permiten examinar que la representación social de la escritura en el proceso de investigación se identifica concretamente con el informe final de resultados (a diferencia de los docentes). Por tanto, buena parte de las dificultades de los procesos escriturales corresponde a este documento. Esta tendencia también se puede rastrear en el syllabus de Práctica Pedagógica III (asignatura destinada exclusivamente a la escritura del trabajo de grado). En este documento se señala que las actividades desarrolladas durante el semestre tienen como objetivo la concreción de la sistematización del trabajo de grado (informe final) seleccionado por el estudiante, sin que se haga explícito secuencias, pautas o estrategias que permitan llegar a él.

En este sentido, se puede identificar que de manera implícita el documento de trabajo de grado realizado por el estudiante se constituye como un producto y no como la suma de las acciones realizadas en el proceso. Perspectiva que contrasta con la concepción de formación de investigación que tiene el programa de LLCI. En relación con los procesos de investigación la Facultad de Educación y de acuerdo con el Proyecto Educativo Institucional (PEI), propone "guiar al estudiante desde las nociones de lo que es la investigación y sus métodos hasta el dominio de las competencias que requiere la investigación básica (...) plasmada en un trabajo de grado o práctica investigativa" (UAN, PEP, 52). Dado lo anterior, la investigación para el programa en LLCI constituye uno de los aspectos medulares de la formación, cuyo objetivo es "conocer y aplicar adecuados métodos de investigación que permitan el desarrollo de las acciones propuestas" (UAN, PEP, 43). Es así como la formación investigativa 
se desarrolla en diferentes cursos, metodologías de enseñanza y trabajo en semilleros. Estos espacios se conciben como los preparatorios que le permitirán al estudiante el desarrollo de sus propuestas de trabajo de grado. En este sentido, los documentos institucionales evidencian referentes teóricos y metodológicos elementales para el desarrollo de procesos investigativos y entienden la realización del trabajo de grado como elemento fundamental en el avance de los objetivos de la investigación.

Es así que la formación investigativa que se propone en el programa LLCI, considera la investigación como un proceso de aprendizaje en todos los ámbitos y momentos de formación estudiantil. En contraste, se puede apreciar en las representaciones sociales de los estudiantes y maestros que la perspectiva de aprendizaje en la investigación se diluye a favor de una que privilegia la comunicación de la información. Por tanto, la escritura no se piensa como una herramienta que ayude a organizar y formular estructuras de pensamiento, sino como un canal comunicativo.

\section{Momentos de la escritura}

Al prevalecer un sistema de creencias sociales que piensan la escritura en la investigación desde una perspectiva comunicativa, en la escritura de trabajos de grado por parte de los estudiantes de LLCI prevalecen las prácticas escriturales al final de los ejercicios y, muy poco, antes y después del informe final. Es así que el $80 \%$ de los estudiantes señala que escribieron apartados de su trabajo de grado cuando lo solicitaba el asesor y para la entrega del informe final. Apenas un 33\% de los encuestados señaló que escribió en el momento de recolectar y analizar la información. Resulta interesante al reflexionar el anterior consolidado, que los estudiantes, de acuerdo al reglamento de trabajo de grado de la Facultad, tienen que entregar un anteproyecto escrito antes de empezar su investigación, y entregar a sus docentes de las asignaturas de práctica pedagógica investigativa avances de la propuesta realizada. Por lo tanto, la contraposición de estas dos situaciones nos permite identificar que algo ocurre para que los estudiantes no reconozcan estos escritos como parte de sus procesos investigativos. Es decir, todos los escritos como borradores, presentaciones y notas realizadas antes del informe final, no se comprenden como parte del proceso de escritura, sino quizá como etapas de los procesos de ciertas asignaturas.

\section{Escritura privada y escritura pública}

De cierta forma, el reconocimiento de la escritura del trabajo de grado con carácter comunicativo produce la sensación de que la escritura en la investigación es una práctica pública. El $90 \%$ de los estudiantes la identifican pública, y tan solo un $25 \%$ como una práctica privada (existía la indicación de poder marcar las dos opciones). Este desconocimiento de la escritura de trabajo de grado como una práctica privada quizá obedece a la ausencia de una escritura enfocada en el aprendizaje y el desarrollo de los procesos cognitivos de los estudiantes. Por tanto, se escribe cuando hay una solicitud externa, cuando hay proceso de evaluación, y no en espacios privados de reconceptualización y comprensión del conocimiento.

\section{Conclusiones}

Las anteriores indagaciones acerca de las representaciones sociales de la escritura en los procesos de formación en investigación en la LLCI de la UAN permiten encontrar contrastes y divergencias acerca de las creencias que dirigen las acciones de los estudiantes y docentes en las prácticas escriturales. Estas consideraciones sobre el saber común, no definitivas, parecen concordar con aquel consenso implícito de la tradición occidental que considera el logos, la palabra escrita, en palabras de Calsamiglia y Tusón (2002), como 
"el logro más extraordinario en la búsqueda de una representación económica y funcional de unidades lingüísticas" (p. 72), que ha permitido acentuar "el poder humano de la abstracción, de reflexión, de aislarse del contexto más inmediato, con lo que resulta un estilo cognitivo que prioriza la actividad intelectual" (p. 73).

A pesar de esta correspondencia inicial entre saber común y pensamiento científico, las divergencias se hacen evidentes cuando se empiezan a revisar los procesos y prácticas que permiten consolidar la escritura en dicha perspectiva. Tal heterogeneidad en momentos no corresponde a una riqueza que entiende la escritura como un objeto con múltiples entradas, sino por el contrario, a la relativización de sus componentes y procesos. Dicha situación se puede observar en las representaciones señaladas, ya que de forma amplia ningún miembro de la comunidad académica discute la importancia e inclusión de la escritura en la educación superior y en los procesos de investigación, pero tales procesos no se revisan en su especificidad de acuerdo con los propósitos epistemológicos y discursivos de cada profesión. En contraste, se reduce la problemática a cursos temáticos que pretenden desarrollar las habilidades de la escritura. La reflexión de la escritura en la universidad, teniendo en cuenta sus fines y posibilidades, no puede ser un elemento ajeno a los procesos desarrollados en los conocimientos disciplinares de cada programa y, mucho menos, seguirse asumiendo desde la obviedad engañosa que asume que como todos la consideran importante, todos son conocedores de sus elementos y procesos.

Por tanto es necesario que en la universidad los procesos de escritura de los estudiantes, como lo señala Linda Flower (cita recogida por Carlino 2006, p. 23), avancen a partir de sus propios procesos cognitivos y sean conscientes de las diferencias entre las distintas culturas escritas, ya que, en palabras de Flower,

Escribir, para los estudiantes, es un acto de cruzar fronteras -o de pararse en el umbral tratando de imaginar cómo cruzar-. Cuando quienes escriben se trasladan del hogar a la escuela, desde la educación inicial a la superior, y de disciplina en disciplina, encuentran una variedad de comunidades discursivas, con sus lenguajes y convenciones especiales, con sus estándares para argumentar y dar razones, y con sus propias historias, como un discurso en el cual se ha formado un conjunto de supuestos, lugares comunes y cuestiones "claves" que los de adentro comparten (p. 23).

El acto de escribir constituye una forma de aprendizaje valiosa, que permite a los estudiantes formar sus propios marcos de conocimiento identificando lugares y conceptos autónomos, que quizá sean difíciles de prever a través de otras formas de aprendizajes. Ocuparse de la escritura implica involucrarse directamente en las estructuras de conocimiento de cada disciplina debido a que "escribir exige poner en relación lo que uno ya sabe con lo que demanda la actual situación de escritura, y que esta puesta en relación no resulta fácil porque implica construir un nexo entre el conocimiento viejo y nuevo" (Carlino, 2006, p. 25). De ahí que cuando escribimos podemos volver a lo pensado cuantas veces sea necesario, proponer, reformular, cambiar, pero lo más importante, producir y crear, encontrar aquellos lugares que no han sido explorados e inventarlos a partir de los límites de lo conocido y nuestros deseos.

Desde esta perspectiva, la escritura constituye un desafío en las prácticas actuales de la educación superior. En síntesis, siguiendo con la propuesta de Carlino (2006), la escritura en la universidad permite la extensión del "conocimiento colectivo y compartido de las disciplinas" (p. 6). Desde este punto de vista, la escritura tiene una función comunicativa esencialmente. En segundo lugar, la escritura permite "otra clase de transformación y reorganización del conocimiento, que se da cuando alguien tiene que reformular lo que ya sabe para una nueva circunstancia" (p. 6). No es solamente comunicar, sino reconceptualizar y reflexionar el conocimiento adquirido. Precisa 
Carlino, se trata de "pensar con la ayuda de la escritura" (p. 6). Sin embargo, los estudiantes, antes que pensar con la escritura, realizan prácticas de escritura como transcripción y corrección gramatical. Señala Sommers (cita recogida por Carlino, 2005a), que ante estas prácticas el docente, a través de la revisión, debe animar al estudiante para que corra el riesgo de cambiar sus ideas hacia lo novedoso: nuestros comentarios deberán alentar en los alumnos una revisión de diferente orden de complejidad y sofisticación respecto de la que por sí mismos identifican, forzándolos a una vuelta al caos (p. 30).

En este sentido, escribir, escribirse, ser sujeto de la escritura, como señala Olson (1998), consiste en revisar cómo el ser humano comprende el mundo y a sí mismo. Tal proceso implica reformular y polemizar los órdenes conceptuales propios en busca de aquellos cambios que generen nuevos órdenes cognitivos:

Desde la perspectiva del que escribe, todo texto "fragua" en algún punto del proceso, endureciéndose con cemento. Más allá de ese punto, cuando lenguaje y pensamiento han perdido maleabilidad, se requiere algo del orden de la dinamita para promover cambios profundos. Los escritores más experimentados dilatan este punto de solidificación y reconsideran y revisan a fondo su trabajo antes de dar por terminado un primer borrador completo. La escritura de los estudiantes, en cambio, tiende a fraguar casi en el momento en que toca el papel (...) Los primeros pensamientos se vuelven los últimos, y los segundos pensamientos parecen destructivos (Carlino, 2006, p. 31).
Para que los primeros pensamientos dejen de ser los últimos en los escritos de los estudiantes, se necesita proponer la reflexión del aprendizaje de la escritura en la universidad desde el reconocimiento de sus posibilidades comunicativas, discursivas y epistémicas; en relación con propuestas didácticas propias de las disciplinas. Se trata de que la cultura escrita permee todo el proceso formativo para potencializar el pensamiento, generar nuevas formas de representación y contraste de la realidad, organizar el conocimiento, resolver problemas, entre otras posibilidades. Pensar con la escritura, resalta Carlino (2006), implica un proceso lento, un proceso "que cuesta porque implica al menos dos renuncias: 1 ) renunciar a la fantasía de poder decir todo lo que uno sabe; 2) renunciar, cuando uno ya escribió, aparte de eso que se ha escrito" (p. 9).

La escritura no es una práctica fácil, ya que cuando escribimos atravesamos "peldaños de una escalera de caracol: avanza lenta y espiralmente" (Carlino, 2006, p. 13). Quizás son el sufrimiento y el dolor los que permiten la escritura verdadera. Solo cuando el papel o el teclado de un dispositivo electrónico tienen las marcas invisibles del sufrimiento y el esfuerzo del escritor, se ha podido transformar el pensamiento y el mundo a través de la escritura. Es en ese momento en el que podemos afirmar, como el profesor de arte de uno de los cuentos del Libro de los amores ridículos de Milan Kundera (1987), que "hay cosas que he conseguido comprender, cuyo sentido he descifrado, cosas a las que quiero y que tomo en serio. $Y$ entonces no se puede bromear" (p. 43).

\section{Referencias}

Arnoux, E., Borsinger, A., Carlino, P., Di Stefano, M., Pereira, C. y Silvestre, A. (2004). La intervención pedagógica en el proceso de escritura de tesis de posgrado. Revista de la Maestría en Salud Pública, 2 (3), 1-16.

Arnoux, E. (2006). Incidencia de la lectura de pares y expertos en la reescritura de tramos del trabajo de tesis. Revista de lingüistica teórica y aplicada, 44 (1), 95-118.

Barthes, R. (1978). Roland Barthes por Roland Barthes (J. Sucre, trad.). Caracas: Monte Avila. Calsamiglia, H. y Tusón, A. (2002). Las cosas del decir. Manual del discurso. Barcelona: Ariel. 
Carlino, P. (2003). La experiencia de escribir una tesis: contextos que la vuelven más difícil. Revista Anales del Instituto de lingüística, 24, (24-26), 41-62.

Carlino, P. (2004a). Culturas académicas contrastantes en Australia, EE. UU. y Argentina: representaciones y prácticas sobre la escritura y sobre la supervisión de tesis en el grado y posgrado universitarios. Mente y Cultura: Cambios representacionales en el aprendizaje. Centro Regional Bariloche de la Universidad Nacional del Comahue y por la Facultad de Psicología de la Universidad Autónoma de Madrid, Universidad Nacional del Comahue.

Carlino, P. (2004b). El proceso de escritura académica: cuatro dificultades de la enseñanza universitaria. Revista Educere, 8 (26), 321-327.

Carlino, P. (2004c). Exploración de géneros, diarios de tesis y revisión entre pares: análisis de un ciclo de investigación-acción en talleres de tesis de posgrado. En E. Arnoux (Comp.), Escritura y producción de conocimiento en carreras de posgrado. Buenos Aires: Santiago Arcos editores.

Carlino, P. (2005a). Escribir, leer y aprender en la universidad. Una introducción a la alfabetización académica. Buenos Aires: FCE.

Carlino, P. (2005b), Revisión entre pares: un proceso y una práctica social que los posgrados pueden enseñar. Ponencia en el panel Análisis del discurso científico, Congreso Nacional de Lingüística, Salta, 48 de junio de 2005. Recuperado de https://sites.google.com/site/giceolem2010/ posgrado

Carlino, P. (2006). La escritura en la investigación. Documentos de trabajo. Escuela de Educación. Buenos Aires: Universidad de San Andrés. Recuperado de https://www.aacademica.org/paula. carlino/66.pdf

Carlino, P. (2013). Alfabetización académica diez años después. Revista Mexicana de Investigación Educativa, 18 (57), 355-381.

Erickson, F. (1989). Métodos cualitativos de investigación sobre la enseñanza. La investigación de la enseñanza. 2: métodos cualitativos y de observación. Barcelona: Paidós, pp. 195-302.

Farr, R. (1985). Las representaciones sociales. En S, Moscovici (Comp.), Psicología social (p, 497). Barcelona: Paidós.

Ferrer, E. (2015). Entrevista a Teun Van Dijk. Revista Papeles, 6 (12-13), 10-11.
Glaser, B. (1992). Basic of Grounded Theory Analysis: Emergence vs. Forcing. Mill Valley: C. A. Sociology Press.

González, B. y Salazar, A. (2015). Formación inicial en lectura y escritura en la universidad. De la educación media al desempeño académico en la educación superior. Bogotá: Pontificia Universidad Javeriana.

Jodelet, D. (1985). La representación social: fenómenos, concepto y teoría. En S, Moscovici (Comp.), Psicología social.Barcelona: Paidós.

Jurado, F. (1998). Investigación, escritura y educación. Bogotá: UNAL-Plaza \& Janes.

Kundera, M. (1987). El libro de los amores ridiculos. (F. Valenzuela, trad.). Barcelona: Tusquets.

Lerner, D. (2001). Leer y escribir en la escuela. Lo real, lo posible, necesario. México: FCE.

Lucca, N. y Berríos, R. (2003). Investigación cualitativa, fundamentos, diseños y estrategias. Colombia: Ediciones S. M.

Moya, C., González, A. y Vanegas I. (2013). Escribir hoy en el posgrado. Escritura académica y producción de conocimiento. Bogotá: Universidad Externado de Colombia.

Olson, D. (1998). El mundo sobre el papel. El impacto de la escritura y la lectura en la estructura del conocimiento. (P. Willson, trad.). Barcelona: Gedisa.

Ortiz, E. (2015). La escritura académica en el contexto universitario. Revista del Instituto de Estudios en Educación Universidad del Norte, 22, 1-16.

Pérez A. y Rincón G. (Ed). (2013) ¿Para qué se lee y se escribe en la universidad Colombiana? un aporte a la consolidación de la cultura académica del país. Bogotá: COLCIENCIAS.

Saby, J. (2012). El rol del docente-investigador en el marco de la Investigación formativa. Revista Papeles, 4 (8), 23-30.

Sánchez, H. (2013). El estudio del currículo en los procesos de formación docente. Revista Caminos Educativos, 2, 93-102.

Universidad Antonio Nariño. (2014). Proyecto Educativo del Programa (PEP) Licenciatura en Lengua Castellana e Inglés. Documento de circulación limitada.

Zuleta, F. (1982). Sobre la lectura. Bogotá: Ministerio de Educación Nacional. Recuperado de http://www.mineducacion.gov.co/cvn/1665/ articles-99018_archivo_pdf.pdf 